\title{
openheart Impact of selected comorbidities on the presentation and management of aortic stenosis
}

\author{
Tanja K. Rudolph, ${ }^{1}$ David Messika-Zeitoun (D) , ${ }^{2}$ Norbert Frey, ${ }^{3}$ \\ Jeetendra Thambyrajah, ${ }^{4}$ Antonio Serra, ${ }^{5}$ Eberhard Schulz, ${ }^{6}$ Jiri Maly, ${ }^{7,8}$ \\ Marco Aiello, ${ }^{9}$ Guy Lloyd, ${ }^{10}$ Alessandro Santo Bortone, ${ }^{11}$ Alberto Clerici, ${ }^{12}$ \\ Georg Delle-Karth, ${ }^{13}$ Johannes Rieber, ${ }^{14}$ Ciro Indolfi, ${ }^{15}$ Massimo Mancone, ${ }^{16}$ \\ Loic Belle, ${ }^{17}$ Alexander Lauten, ${ }^{18}$ Martin Arnold, ${ }^{19}$ Berto J Bouma, ${ }^{20}$ Matthias Lutz, ${ }^{3}$ \\ Cornelia Deutsch, ${ }^{21}$ Jana Kurucova, ${ }^{22}$ Martin Thoenes, ${ }^{23}$ Peter Bramlage (D) ,21 \\ Richard P. Steeds ${ }^{24}$
}

\begin{abstract}
- Additional material is published online only. To view please visit the journal online (http://dx.doi.org/10.1136/ openhrt-2020-001271).
\end{abstract}

To cite: Rudolph TK, Messika-Zeitoun D, Frey N, et al. Impact of selected comorbidities on the presentation and management of aortic stenosis. Open Heart 2020;7:e001271. doi:10.1136/ openhrt-2020-00127

Received 19 February 2020 Revised 26 March 2020 Accepted 2 June 2020
Check for updates

(C) Author(s) (or their employer(s)) 2020. Re-use permitted under CC BY-NC. No commercial re-use. See rights and permissions. Published by BMJ.

For numbered affiliations see end of article.

\section{Correspondence to}

Professor Tanja K. Rudolph; tk. rudolph@me.com

\section{ABSTRACT}

Background Contemporary data regarding the impact of comorbidities on the clinical presentation and management of patients with severe aortic stenosis (AS) are scarce.

Methods Prospective registry of severe patients with AS across 23 centres in nine European countries.

Results Of the 2171 patients, chronic kidney disease (CKD 27.3\%), left ventricular ejection fraction (LVEF) $<50 \%$ (22.0\%), atrial fibrillation $(15.9 \%)$ and chronic obstructive pulmonary disease $(11.4 \%)$ were the most prevalent comorbidities $(49.3 \%$ none, $33.9 \%$ one and $16.8 \% \geq 2$ of these). The decision to perform aortic valve replacement (AVR) was taken in a comparable proportion (67\%, $72 \%$ and $69 \%$, in patients with 0,1 and $\geq 2$ comorbidities; $\mathrm{p}=0.186$ ). However, the decision for TAVI was more common with more comorbidities $35.4 \%, 54.0 \%$ and $57.0 \%$ for $n 0,1$ and $\geq 2 ; p<0.001$ ), while the decision for surgical AVR (SAVR) was decreased with increasing comorbidity burden $(31.9 \%, 17.4 \%$ and $12.3 \%$; $p<0.001)$. The proportion of patients with planned AVRs that were performed within 3 months was significantly higher in patients with 1 or $\geq 2$ comorbidities than in those without $(8.7 \%, 10.0 \%$ and $15.7 \% ; p<0.001)$. Furthermore, the mean time to AVR was significantly shorter in patients with one (30.5 days) or $\geq 2$ comorbidities ( 30.8 days) than in those without ( 35.7 days; $p=0.012$ ). Patients with reduced LVEF tended to be offered an AVR more frequently and with a shorter delay while patients with CKD were less frequently treated.

Conclusions Comorbidities in severe patients with AS affect the presentation and management of patients with severe AS. TAVI was offered more often than SAVR and performed within a shorter time period.

\section{INTRODUCTION}

Aortic stenosis (AS) is the most frequent native valve disease worldwide, affecting approximately $3.4 \%$ of elderly individuals in Europe and the USA. ${ }^{12}$ Patients with severe AS who do not undergo timely aortic valve

\section{Key questions}

What is already known about this subject?

- Current European guidelines recommend that aortic valve replacement (AVR) be performed in patients with aortic stenosis (AS) that are symptomatic, experience symptoms/hypotension during exercise testing and/or that have a left ventricular ejection fraction (LVEF) $<50 \%$. Contemporary data regarding the impact of comorbidities on the clinical presentation and management of patients with severe AS are scarce.

What does this study add?

- IMPULSE is a prospective, multinational registry of patients with AS across Europe. The present data suggest an overall relationship between the presence of chronic kidney disease (CKD), reduced LVEF, atrial fibrillation and chronic obstructive pulmonary disease with the presentation of patients with severe AS and their management. Certain comorbidities appear to have more of an influence over treatment decisions, with CKD an apparent deterrent and LVEF $<50 \%$ an apparent incentive for AVR.

How might this impact on clinical practice?

- The life-saving AVR- intervention was still denied in a high proportion of patients, with many of the planned AVRs not carried out within 3 months. On a more encouraging note, transcatheter aortic valve implantation was more frequently assigned to comorbid patients, with such interventions performed within a shorter time period.

replacement (AVR) face an extremely poor prognosis; this is particularly true following symptom onset, at which point average survival is just 2-3 years. ${ }^{3}$ Consequently, rapid, effective management is paramount.

Current European guidelines recommend that AVR be performed in patients with AS 
that are symptomatic, experience symptoms/hypotension during exercise testing, and/or that have a left ventricular ejection fraction (LVEF) $<50 \% .{ }^{4}$ However, despite the ever-growing availability of transcatheter aortic valve implantation (TAVI) as a minimally invasive alternative to surgical AVR (SAVR), studies suggest that up to $40 \%$ of patients with severe AS still do not receive either of these life-saving interventions. ${ }^{5-7}$ The reason for this appears to be multifactorial, though a high comorbidity burden has been suggested as a key driver. ${ }^{8}$ Furthermore, certain comorbidities may have clinical presentations resembling those of AS. To the best of our knowledge, no formal investigations into such effects have been carried out to date.

The aim of this analysis therefore was to assess the impact of comorbidities on disease presentation and management decisions in patients with severe AS.

\section{METHODS}

The design and rationale of IMPULSE, a prospective, multinational registry of patients with AS across Europe, has been previously described. ${ }^{69}$ Briefly, the study formed part of a quality of care initiative combining observational and interventional elements. In total, patients were enrolled at 23 centres across nine countries (Austria, Czech Republic, France, Germany, Italy, the Netherlands, Spain, Switzerland and the UK) up until April 2017; all sites offered the full range of treatment options for AS. Informed consent was obtained prior to patient enrolment.

\section{Patients}

Patients aged at least 18 years that had been referred for echocardiography and were diagnosed with severe AS (defined as aortic valve area (AVA) $<1 \mathrm{~cm}^{2}$, indexed AVA $<0.6 \mathrm{~cm}^{2} / \mathrm{m}^{2}$, maximum jet velocity $\left(\mathrm{V}_{\max }\right)>4 \mathrm{~m} / \mathrm{s}$ or mean transvalvular gradient $>40 \mathrm{~mm} \mathrm{Hg}$ ) symptomatic or asymptomatic were consecutively enrolled. We excluded patients that had previously undergone AVR.

\section{Data collection and definitions}

At baseline, we documented data regarding patient demographics, medical history and symptoms (chest pain, shortness of breath and dizziness on exertion/ syncope) in an electronic case report form. Angina (Canadian Cardiovascular Society class III or IV), New York Heart Association (NYHA) class III/IV and dizziness on exertion/syncope were considered severe symptoms. Details of each patient's AVA, mean AV gradient, concomitant mitral/tricuspid regurgitation, LVEF and left ventricle dimensions were recorded from their echocardiogram. All treatment decisions (TAVI, SAVR, balloon aortic valvuloplasty (BAV), watchful waiting and active decision not to treat) were documented. All data were subject to automatic checks for plausibility and completeness.

\section{Statistics}

As we aimed to assess the impact of comorbidities on disease presentation and management decisions, we described the prevalence of and identified the four most prevalent comorbidities. Patients were then stratified according to baseline comorbidity burden. Three groups were built, according to the absence, the presence of one or the presence of at least two of the following four comorbidities: LVEF $<50 \%$; chronic kidney disease (CKD; defined as creatinine clearance $<50 \mathrm{~mL} / \mathrm{min}$ ), atrial fibrillation (AF; based on physician diagnosis) and chronic obstructive pulmonary disease (COPD; defined as per EuroSCORE (ES)). Patients were also grouped according to the presence/absence of each of these individual comorbidity types. To serve as a surrogate for overall comorbidity, patients were also divided into those with/without a high surgical risk score (defined as ES $\mathrm{I} \geq 10 \%$ and/or $\mathrm{ES} \mathrm{II} \geq 4 \%$ ).

Data were analysed using descriptive statistics, with continuous variables presented as means with $\mathrm{SD}$ and categorical variables as absolute values with percentages. Comparisons across comorbidity-burden groups were made using analysis of variance or Kruskal-Wallis test for continuous and Pearson's $\mathrm{X}^{2}$ for categorical variables. Comparisons between patients with/without a particular comorbidity type were made using a Pearson's $\mathrm{X}^{2}$ or Fisher's exact test for categorical variables and a t-test or Wilcoxon signed-rank test for continuous variables. The association between each comorbidity of interest and the presentation of AS symptoms was explored using univariate logistic regression analysis and presented as ORs with 95\% CIs. All statistical analyses were performed using SPSS V.24.0 (IBM), with a $\mathrm{p}<0.05$ considered statistically significant.

\section{RESULTS}

Of the 2171 patients documented, the mean age was 77.9 years, $48.0 \%$ were female and $80.3 \%$ were symptomatic (51.7\% severe). The most common comorbidities were CKD (27.3\%), a reduced LVEF $<50 \%$ (22.0\%), $\mathrm{AF}(15.9 \%)$ and COPD (11.4\%), representing $50.7 \%$ $(\mathrm{n}=1100)$ of all patients (figure 1). While 1071 patients $(49.3 \%)$ had none of the comorbidities of interest, 735 (33.9\%) had one comorbidity, and 365 (16.8\%) had two comorbidities or more. A high surgical risk defined as an ES I $\geq 10 \%$ and/or ES II $\geq 4 \%$ was documented for $42.9 \%$ of the patients.

\section{Comorbidity and as presentation}

When patients were stratified by the number of comorbidities, there was an increase in age (range mean 75.7 up to 81.4 years), the proportion of severely frail patients (2.5 up to $10.8 \%$ ), and the surgical risk (ES II mean 2.2 up to $8.2 \%$ ) (table 1). Furthermore, mitral (mean 5.2 up to $20.4 \%$ ) as well as tricuspid (3.9 up to $18.9 \%$ ) regurgitation was more prevalent. Similar trends were observed when comparing patients without a particular comorbidity of 


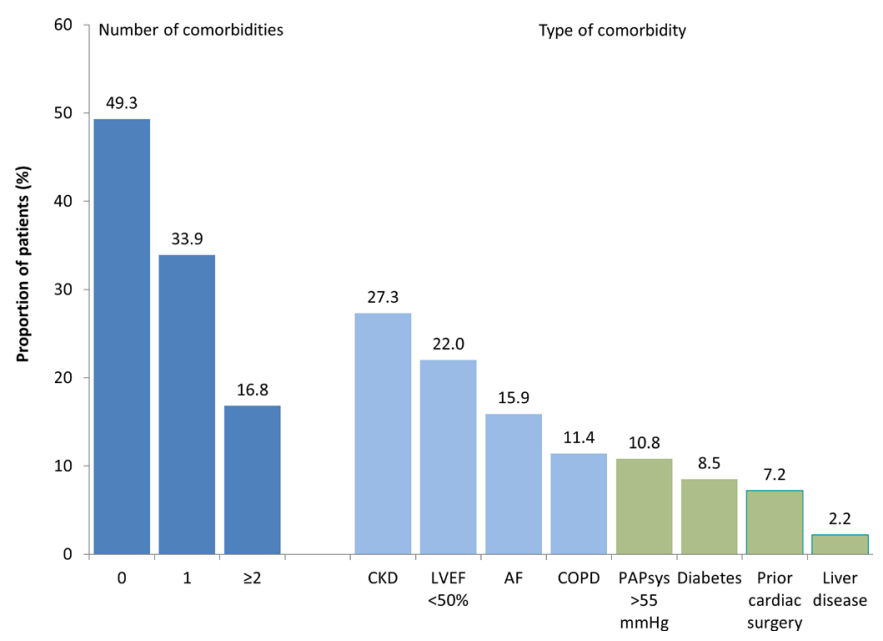

Figure 1 Prevalence of comorbidities. AF, atrial fibrillation; CKD, chronic kidney disease; COPD, chronic obstructive pulmonary disease; LVEF, left ventricular ejection fraction; light blue bars, those considered for the comorbidity burden in dark blue; green bars, those not considered for the comorbidity burden in dark blue.

interest to those with the particular comorbidity (online supplementary tables 1-5). One exception was gender; compared with patients without the respective comorbidity, females were more common among patients with CKD $(55.6 \%$ vs $45.1 \%$; $\mathrm{p}<0.001)$, while males were more common among those with an LVEF $<50 \%(63.6 \%$ vs $49.1 \%$; $\mathrm{p}<0.001)$.

The proportion of patients with any symptoms $(73.6 \%$, $84.4 \%, 91.8 \% ; \mathrm{p}<0.001)$ and severe symptoms $(40.8 \%$, $58.4 \%$ and $70.1 \%$; $\mathrm{p}<0.001)$ increased alongside comorbidity burden (table 1). Furthermore, each of the single comorbidities of interest was significantly associated with a greater likelihood of being symptomatic (table 2). Reduced EF and COPD were strongly associated with any as well as severe symptoms ( $\mathrm{OR}>2.0)$ while there was a lesser association of $\mathrm{CKD}$ and $\mathrm{AF}$ with any symptom class $(\mathrm{OR}>1.0$ to $<2.0)$. Of note, a high ES was associated with symptoms throughout all domains whether it be any (OR 2.55 ; $95 \%$ CI 1.9 to 3.31 ) or severe (OR 2.27; $95 \%$ CI 1.88 to 2.74 ) symptoms and the only who was associated with chest pain (OR 1.66; 95\% CI 1.33 to 2.07). So both, general and cardiac presentation were worse in patients with comorbidities.

\section{Treatment decisions according to comorbidity burden}

The decision to perform AVR was taken in comparable proportions of patients, regardless of comorbidity burden $(69.0 \%$ overall), with $31 \%$ not assigned to AVR (figure 2). However, the decision to perform TAVI was more common in patients with a greater number of comorbidities $(35.4 \%, 54.0 \%$ and $57.0 \%$ for patients with no, one and at least two comorbidities, respectively; $\mathrm{p}<0.001$ across groups), while the decision for SAVR was decreased with increasing comorbidity burden $(31.9 \%$, $17.4 \%$, and $12.3 \% ; \mathrm{p}<0.001$ across groups). The same trends were observed when the analysis was confined to symptomatic patients only with $41.8 \%, 57.4 \%$ and $58.8 \%$ of patients being assigned to TAVI and $36.5 \%, 18.0 \%$ and $13.0 \%$ being assigned to SAVR $(\mathrm{p}<0.001)$. An active decision not to treat $(5.8 \%, 9.5 \%$ and $12.6 \% ; \mathrm{p}<0.001)$ was more frequently chosen in those with a higher comorbidity burden.

The proportion of patients with planned AVRs that were performed within 3 months was significantly higher in patients with one or at least two comorbidities than in those with no comorbidities $(15.7 \%, 10.0 \%$ and $8.7 \%$ respectively; $\mathrm{p}<0.001$ ) (figure 2 ). This was mostly observed based on the rates of non-performance in patients scheduled to undergo surgery and none of the comorbidities of interest $(p<0.001)$, while there was no such trend among patients scheduled for TAVI.

Furthermore, the mean time to AVR was significantly shorter in patients with one $(30.5 \pm 33.6)$ or at least two comorbidities (30.8 \pm 32.3 days) than in those without (35.7 \pm 36.1 days; $\mathrm{p}=0.012$ across groups) (table 1 ).

\section{Treatment decisions according to comorbidity type}

The decision to perform AVR was taken in comparable proportions of patients with and without $\mathrm{AF}$, and those with and without COPD. This choice was, however, significantly more frequent in patients with an LVEF $\leq 50 \%$ compared with those with an LVEF $>50 \%$ (78.3\% vs $66.4 \% ; \mathrm{p}<0.001)$ and significantly less frequent in those with CKD compared with those without CKD $(65.1 \%$ vs $71.4 \% ; \mathrm{p}=0.011$ ) (figure 2). In general, TAVI was a more common treatment choice in patients with compared with without each of the comorbidities of interest, with the inverse true for SAVR.

Performance of a planned AVR within 3 months of the treatment decision was more common in patients with an LVEF $\leq 50 \%$ compared with those with an LVEF $>50 \%$ ( $68.1 \%$ vs $53.4 \%$; $\mathrm{p}<0.001)$. The mean time to AVR was significantly shorter in patients with CKD compared with those without this condition $(29.0 \pm 29.2$ vs $34.3 \pm 35.7$; $\mathrm{p}=0.013$ ) (online supplementary tables 1-5). These trends were not observed for AF or COPD.

\section{DISCUSSION}

In our contemporary cohort of patients with severe AS, renal impairment, impaired $\mathrm{LV}$ function, $\mathrm{AF}$ and $\mathrm{COPD}$ were common. Each was found to be associated with (severe) symptoms. Though the number of comorbidities did not appear to affect the overall decision to perform AVR, TAVI was more frequently assigned to patients with more comorbidities, with interventions being performed within a shorter time period. Specifically, CKD appeared to be a deterrent for AVR, with LVEF $\leq 50 \%$ an apparent incentive. Overall, nearly one-third of patients were not assigned to undergo life-saving AVR, with this value being as high as $35 \%$ in patients with CKD in the present registry.

\section{Comorbidity burden}

Approximately half of the patients with severe AS enrolled in the IMPULSE registry had one or more of the 
Table 1 Baseline patient characteristics, echocardiographic parameters and treatment decisions by number of comorbidities

\begin{tabular}{|c|c|c|c|c|c|}
\hline & $\begin{array}{l}\text { All patients } \\
(n=2171)\end{array}$ & $\begin{array}{l}0 \text { comorbidities* } \\
(n=1071)\end{array}$ & $\begin{array}{l}\text { One comorbidity* } \\
(n=735)\end{array}$ & $\begin{array}{l}\geq 2 \text { comorbidities* } \\
(n=365)\end{array}$ & $\begin{array}{l}\text { P value } \\
\text { across } \\
\text { comorbidity } \\
\text { groups }\end{array}$ \\
\hline Age (years) & $77.9 \pm 10.0$ & $75.7 \pm 10.7$ & $79.4 \pm 9.0$ & $81.4 \pm 7.8$ & $<0.001$ \\
\hline Female gender & 48.0 & 48.0 & 50.2 & 43.3 & 0.097 \\
\hline Severe frailty† & 5.1 & 2.5 & 6.1 & 10.8 & $<0.001$ \\
\hline EuroSCORE I (\%) & $15.6 \pm 13.9$ & $9.5 \pm 8.2$ & $17.9 \pm 12.8$ & $26.8 \pm 18.5$ & $<0.001$ \\
\hline EuroSCORE II (\%) & $4.0 \pm 5.0$ & $2.2 \pm 3.0$ & $4.7 \pm 4.7$ & $8.2 \pm 7.2$ & $<0.001$ \\
\hline
\end{tabular}

Echocardiographic valve

parameters

\begin{tabular}{|c|c|c|c|c|c|}
\hline Indexed AV area $\left(\mathrm{cm}^{2} / \mathrm{m}^{2}\right)$ & $0.40 \pm 5.0$ & $0.41 \pm 0.11$ & $0.39 \pm 0.10$ & $0.37 \pm 0.11$ & $<0.001$ \\
\hline $\begin{array}{l}\text { Mean AV gradient (mm } \\
\mathrm{Hg})\end{array}$ & $47.1 \pm 14.7$ & $50.0 \pm 13.3$ & $45.4 \pm 14.8$ & $42.2 \pm 16.3$ & $<0.001$ \\
\hline $\begin{array}{l}\text { Mitral regurgitation grade } \\
\text { III/IV }\end{array}$ & 10.7 & 5.2 & 13.6 & 20.4 & $<0.001$ \\
\hline $\begin{array}{l}\text { Tricuspid regurgitation } \\
\text { grade III/IV }\end{array}$ & 7.9 & 3.9 & 8.1 & 18.9 & $<0.001$ \\
\hline Indexed LV mass $\left(\mathrm{g} / \mathrm{m}^{2}\right)$ & $132.6 \pm 40.9$ & $126.7 \pm 38.0$ & $134.4 \pm 41.1$ & $147.6 \pm 44.7$ & $<0.001$ \\
\hline Symptomatic status $\ddagger$ (\%) & 80.3 & 73.6 & 84.4 & 91.8 & $<0.001$ \\
\hline $\begin{array}{l}\text { Severe symptomatic status§ } \\
(\%)\end{array}$ & 51.7 & 40.8 & 58.4 & 70.1 & $<0.001$ \\
\hline Decision to perform AVR & 69.0 & 67.2 & 71.5 & 69.3 & 0.186 \\
\hline $\begin{array}{l}\text { AVR performed within } \\
3 \text { months }\end{array}$ & 56.3 & 51.5 & 61.5 & 60.5 & $<0.001$ \\
\hline Time to intervention (days) & $33.0 \pm 34.7$ & $35.7 \pm 36.1$ & $30.5 \pm 33.6$ & $30.8 \pm 32.3$ & 0.012 \\
\hline Decision to perform TAVI & 45.0 & 35.4 & 54.0 & 57.0 & $<0.001$ \\
\hline $\begin{array}{l}\text { TAVI performed within } \\
3 \text { months }\end{array}$ & 38.0 & 28.3 & 47.0 & 50.5 & $<0.001$ \\
\hline Time to intervention (days) & $29.2 \pm 34.2$ & $30.0 \pm 36.4$ & $29.2 \pm 33.6$ & $27.5 \pm 31.0$ & 0.755 \\
\hline Decision to perform SAVR & 24.0 & 31.9 & 17.4 & 12.3 & $<0.001$ \\
\hline $\begin{array}{l}\text { SAVR performed within } \\
3 \text { months }\end{array}$ & 18.3 & 23.3 & 14.4 & 10.0 & $<0.001$ \\
\hline Time to intervention (days) & $40.7 \pm 34.5$ & $42.4 \pm 34.7$ & $34.5 \pm 33.4$ & $46.5 \pm 34.4$ & 0.037 \\
\hline Decision not to perform AVR & 27.6 & 29.1 & 25.7 & 26.5 & $<0.001$ \\
\hline
\end{tabular}

*LVEF $<50 \%$, CKD, AF or COPD.

†Defined as an inability to walk $5 \mathrm{~m}$ in $\leq 6 \mathrm{~s}$ and/or to perform activities of daily living. ${ }^{24}$

‡Defined as chest pain, shortness of breath and/or dizziness on exertion/syncope.

§Defined as CCS class III or IV angina, NYHA class III/IV and/or dizziness on exertion/syncope.

AF, atrial fibrillation; AV, aortic valve; AVA, aortic valve area; AVR, aortic valve replacement; CCS, Canadian Cardiovascular Society; CKD, chronic kidney disease ; COPD, chronic obstructive pulmonary disease; LV, left ventricular; LVEF, left ventricular ejection fraction; NYHA, New York Heart Association; SAVR, surgical AVR; TAVI, transcatheter aortic valve implantation .

comorbidities of interest, with a sixth having two or more. In line with the observations of prior studies, those with a greater number of comorbidities were older and more commonly frail. ${ }^{10}$ They also had worse left ventricular function accompanied by lower AV gradients but smaller indexed effective orifice area, indicating a certain proportion of patients with 'low-gradient' AS. ${ }^{11}$ The predictive value of effective orifice area and $\mathrm{LV}$ parameters for symptom onset has been previously reported. ${ }^{12}{ }^{13}$ As such, the observed increase in the prevalence of symptoms alongside increasing comorbidity burden is unsurprising. Nevertheless, the decision to perform AVR was taken in a similar proportion of patients with 0,1 or at least 2 comorbidities.

This likely reflects a balance between the lower disease severity in patients with fewer comorbidities, increasing the tendency towards watchful waiting, and the deterring influence of a higher age and greater surgical risk in those with a higher comorbidity burden, increasing the frequency of decisions not to treat the patient or to 


\begin{tabular}{ccllll}
\hline Table 2 & Association of the most frequent comorbidities with AS symptoms and severity & & \\
\hline & $\begin{array}{l}\text { CKD } \\
(\mathbf{n = 2 1 7 1 )}\end{array}$ & $\begin{array}{l}\text { Low LVEF } \\
(\mathbf{n = 1 0 7 1 )}\end{array}$ & $\begin{array}{l}\text { AF } \\
(\mathbf{n}=735)\end{array}$ & $\begin{array}{l}\text { COPD } \\
(\mathbf{n = 3 6 5 )}\end{array}$ & $\begin{array}{l}\text { High ES } \\
(\mathbf{n = 7 9 5 )}\end{array}$ \\
\hline Symptomatic status & $1.99(1.50-2.65)$ & $2.58(1.85-3.58)$ & $1.45(1.04-2.02)$ & $2.85(1.80-4.52)$ & $2.55(1.97-3.31)$ \\
\hline Chest pain & $1.10(0.87-1.39)$ & $1.25(0.98-1.59)$ & $0.98(0.73-1.31)$ & $1.26(0.92-1.73)$ & $1.66(1.33-2.07)$ \\
\hline Shortness of breath & $1.91(1.49-2.46)$ & $2.99(2.20-4.05)$ & $1.53(1.13-2.07)$ & $3.25(2.12-4.97)$ & $2.53(2.01-3.19)$ \\
\hline Dizziness/syncope & $1.19(0.93-1.51)$ & $1.53(1.20-1.97)$ & $1.33(1.00-1.77)$ & $1.49(1.09-2.05)$ & $1.48(1.19-1.85)$ \\
\hline Severe symptomatic status* & $1.98(1.62-2.44)$ & $2.39(1.92-2.98)$ & $1.49(1.17-1.90)$ & $2.06(1.56-2.73)$ & $2.27(1.88-2.74)$ \\
\hline Angina class III/IV & $2.33(1.47-3.70)$ & $1.58(0.98-2.55)$ & $0.94(0.50-1.75)$ & $1.46(0.80-2.69)$ & $2.13(1.34-3.40)$ \\
\hline NYHA class III/IV & $2.26(1.85-2.78)$ & $2.77(2.23-3.44)$ & $1.43(1.12-1.83)$ & $2.34(1.78-3.08)$ & $2.67(2.20-3.24)$ \\
\hline
\end{tabular}

*Includes dizziness/syncope, as listed under symptomatic status.

$\mathrm{AF}$, atrial fibrillation; CKD, chronic kidney disease; COPD, chronic obstructive pulmonary disease; ES, EuroSCORE; LVEF, left ventricular ejection fraction; NYHA, New York Heart Association.
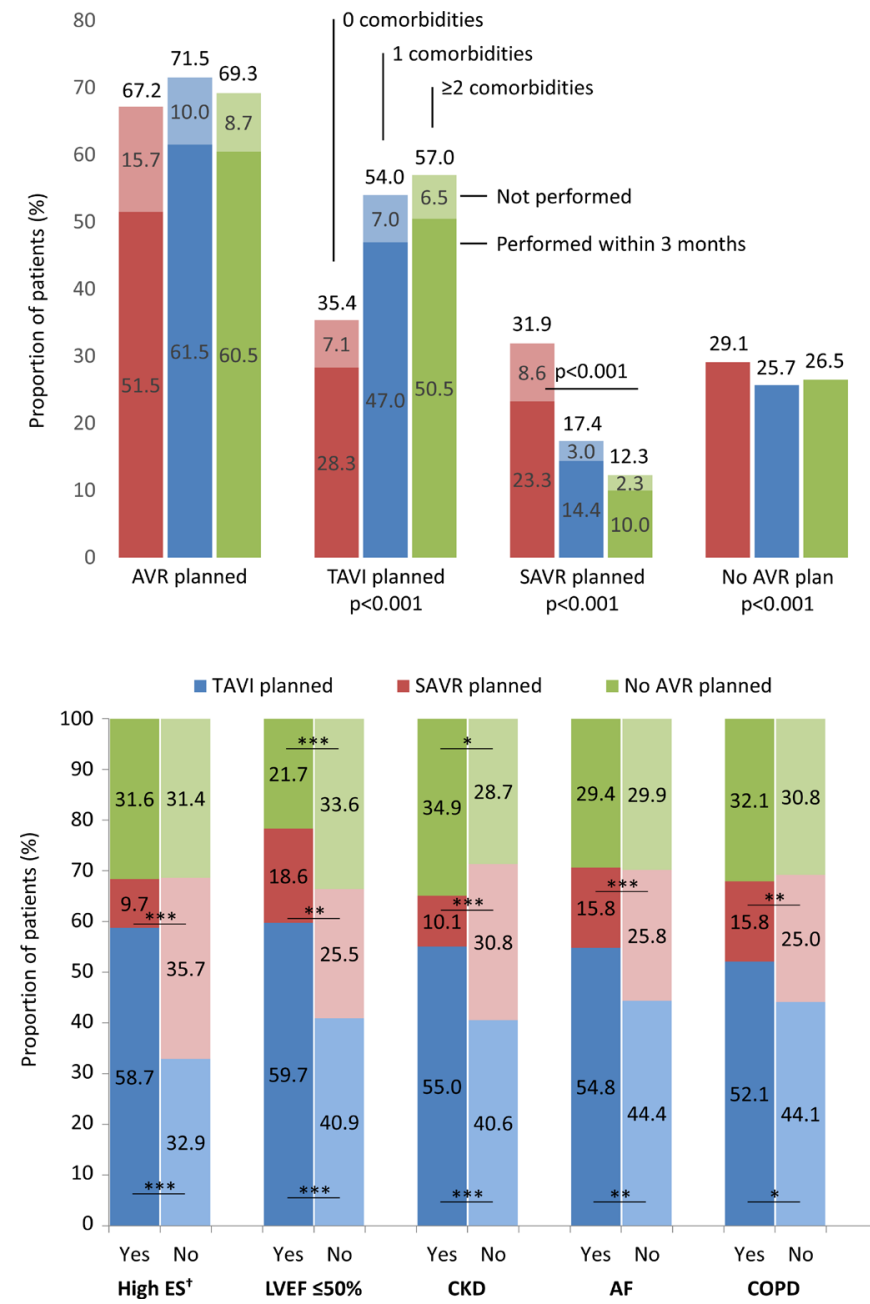

Figure 2 Treatment decision by number and type of comorbidities. $\mathrm{P}<0.001$ for TAVI planned overall, SAVR planned overall and no AVR planned overall; $\mathrm{p}<0.001$ for AVR planned but not performed and SAVR planned but not performed; ${ }^{\dagger} \mathrm{ES} I \geq 10 \%$ or ES II $\geq 4 \%$. ${ }^{*} \mathrm{P}<0.05$; ${ }^{* *} \mathrm{p}<0.01 ;{ }^{* * *} \mathrm{p}<0.001$. AF, atrial fibrillation; AVR, aortic valve replacement; $\mathrm{BAV}$, balloon aortic valvuloplasty; CKD, chronic kidney disease; COPD, chronic obstructive pulmonary disease; LVEF, left ventricular ejection fraction; ES, EuroSCORE; SAVR, surgical aortic valve replacement; TAVI, transcatheter aortic valve implantation; perform BAV instead. Given that guidelines recommend against the performance of AVR in patients with severe comorbidities when it is unlikely to improve quality of life or survival, ${ }^{4}$ the latter trend may be appropriate. However, the observed reluctance to perform AVR in one-third of patients with none of the comorbidities of interest, who had severe AS and a less-than-high surgical risk may indicate a tendency towards undertreatment. ${ }^{4}$ Even in the subset of these latter patients that were assigned to AVR, nearly a quarter did not undergo the procedure within 3 months. Furthermore, the overall rate of patients denied AVR was approximately comparable to that reported by the Euro Heart Survey in 2005, before the advent of TAVI. Encouragingly, TAVI was assigned more often to patients with higher comorbidity burdens, with more SAVR planned in those with fewer comorbidities. This is in line with current guidelines, ${ }^{4}$ and reflects the widespread recognition of TAVI as a safer alternative to SAVR in patients at higher surgical risk.

\section{Chronic kidney disease}

CKD was a highly prevalent comorbidity in the IMPULSE population, affecting over a quarter of patients. In addition to predicting overall symptomatic or severe symptomatic status, CKD was the only comorbidity of interest that was significantly associated with angina class III/IV. The relationship between renal impairment and coronary artery disease (CAD) is well established, explained by their numerous shared risk factors. ${ }^{14}$ However, the principal mechanism underlying angina in AS is increased left ventricular demand, rather than coronary artery stenosis. ${ }^{15}$ Consequently, angina may have been interpreted as a symptom of CAD in patients with CKD, rather than of AS. Whether right or wrong, this is likely to have impacted on treatment decision; speculatively, it is possible that some patients with CKD may have been denied TAVI due to misattribution of AS-related angina symptoms to CAD. This merits further investigation.

CKD appeared to be a deterrent for AVR, which was assigned significantly less frequently in patients with this comorbidity. The observed trend may be justified in the context of the aforementioned benefit considerations 
stipulated by guidelines. ${ }^{4}$ Regardless, a striking preference for TAVI over SAVR was evident in CKD patients, with only $15 \%$ of those scheduled for AVR assigned to SAVR. There is clear evidence that TAVI patients have higher rates of all-cause mortality and major cardiac/ cerebrovascular events, while the SAVR patients had a higher risk of dialysis. Thus, the decision to perform any type of AVR in patients with CKD must be made with extreme caution, with distinct risks associated with the surgical and transcatheter interventions. The appropriacy of the strong preference for TAVI in the present patients with CKD requires further investigation, though this treatment decision might be mainly driven by the strikingly high ES values recorded in this subset.

\section{Left ventricular ejection fraction}

Just over a fifth of patients had an LVEF $<50 \%$. In line with previously reported trends, a larger proportion of these were male. ${ }^{16}$ As with the other comorbidities of interest, a reduced LVEF was predictive of overall symptomatic status, shortness of breath and NYHA class III/IV, but was also one of the only comorbidities specifically predictive for dizziness on exertion/syncope. This is consistent with the idea that patients with impaired LVEFs are less tolerant to rapid heart rhythms..$^{17}$ Given that LVEF $<50 \%$ is an independent criterion for AVR according to European guidelines, ${ }^{4}$ it is no surprise that the choice to perform AVR was significantly more common in patients with a low compared with a preserved LVEF. These individuals were also assigned to TAVI much more frequently with this decision reinforced by the increased surgical risk and by the evidence for a more rapid and complete recovery of LV function after TAVI compared with SAVR. ${ }^{18}$ As such, LVEF appears to have a strong and logical influence on both AS symptoms and treatment decisions.

\section{Chronic obstructive pulmonary disease}

COPD was present in approximately $11 \%$ of patients. While the majority of the trends in patient characteristics across increasing comorbidity burdens were also observed for patients without compared with with COPD, the values for echocardiographic parameters were strikingly similar between these two subsets, indicating similar AS stages. This similarity is largely consistent with another study that reported baseline data for patients with AS stratified by the presence/absence of COPD. ${ }^{19}$ As such, the observed higher likelihood of dizziness on exertion/syncope associated with COPD is probably attributable to the lung disease itself, rather than as a direct consequence of AS. ${ }^{20}$ Furthermore, the fact that AVR was performed in approximately equivalent proportions of patients with/without COPD may also be explained by largely comparable heart disease states. Nevertheless, TAVI was again favoured in patients with COPD, consistent with the higher overall ES in those with this comorbidity.

\section{Atrial fibrillation}

Though AF was a fairly common comorbidity among IMPULSE patients, it appeared to have very little influence on the presentation and management of severe AS. This may be surprising, given that AF has been associated with a higher risk of all-cause and cardiac mortality in patients undergoing TAVI. ${ }^{21} 22$ However, a recent study suggests that the protective effect of AVR is relatively stronger in patients with AF than in those with normal sinus rhythm. ${ }^{23}$ Thus, it is reasonable that the comorbidity is not a direct deterrent.

\section{Limitations}

The major limitation of the present analysis was the lack of adjustment for confounding factors, including other comorbidities that may have influenced AS presentation and treatment decisions. As such, unequivocal identification of independent associations between the comorbidities of interest and symptoms/management strategies is problematic. Nevertheless, the consistency of the observed trends with published data and clinical logic supports the value of our findings, which form a strong basis for further exploration.

\section{CONCLUSIONS}

The present data suggest an overall relationship between the presence of CKD, reduced LVEF, AF and COPD with the presentation of patients with severe AS and their management. Certain comorbidities appear to have more of an influence over treatment decisions, with CKD an apparent deterrent and $\mathrm{LVEF}<50 \%$ an apparent incentive for AVR. However, this life-saving intervention was still denied in a high proportion of patients, with many of the planned AVRs not carried out within 3 months. On a more encouraging note, TAVI was more frequently assigned to comorbid patients, with such interventions performed within a shorter time period.

\section{Author affiliations}

${ }^{1}$ Department of Cardiology, Heart and Diabetes Center Bad Oeynhausen, RuhrUniversity of Bochum, Bad Oeynhausen, Germany

${ }^{2}$ University of Ottawa Heart Institute, Ottawa, Ontario, Canada

${ }^{3}$ Department of Cardiology and Angiology, University of Kiel, Kiel, Germany

${ }^{4}$ James Cook University Hospital, Middlesbrough, UK

${ }^{5}$ Interventional Cardiology Unit, Hospital de la Santa Creu i Sant Pau, Barcelona, Spain

${ }^{6}$ Cardiology Department, AKH Celle, Celle, Germany

${ }^{7}$ Department of Cardiovascular Surgery, Institute for Clinical and Experimental Medicine, Prague, Czech Republic

${ }^{8}$ Department of Cardiovascular Surgery, Second Faculty of Medicine, Charles University, Prague, Czech Republic

${ }^{9}$ Department of Cardiothoracic Surgery, Foundation IRCCS Policlinico S.Matteo, Pavia, Italy

${ }^{10}$ St Bartholomew's Hospital, London, UK

${ }^{11}$ University of Bari, Bari, Italy

${ }^{12}$ University of Turin, Turin, Italy

${ }^{13} 4$ th Medical Department, Hietzing Hospital, Vienna, Austria

${ }^{14}$ Herzkatheterlabor Nymphenburg and Department of Cardiology, University of Munich, Munich, Germany

${ }^{15}$ Division of Cardiology and URT CNR of IFC, Magna Graecia University, Catanzaro, Italy 
${ }^{16}$ Sapienza University of Rome, Rome, Italy

${ }^{17}$ Centre Hospital d'Annecy, Annecy, France

${ }^{18}$ German Centre for Cardiovascular Research (DZHK), University Heart Center \& Charité, Berlin, Germany

${ }^{19}$ Department of Cardiology, University of Erlangen, Erlangen, Germany

${ }^{20}$ University of Amsterdam, Amsterdam, Netherlands

${ }^{21}$ Institute for Pharmacology and Preventive Medicine, Cloppenburg, Germany

${ }^{22}$ Edwards Lifesciences, Prague, Czech Republic

${ }^{23}$ Edwards Lifesciences, Nyon, Switzerland

${ }^{24}$ Queen Elizabeth Hospital \& Institute of Cardiovascular Sciences, University of Birmingham, Birmingham, UK

\section{Twitter Richard P. Steeds @Richard.Steeds}

Acknowledgements Data were captured using the s4trials Software provided by Software for Trials Europe, Berlin, Germany.

Contributors NF, RPS, DM-Z, JK, MT and PB were involved in the conception and design of the study. PB and TKR drafted the manuscript and all other authors revised the article for important intellectual content. All authors gave final approval of the version.

Funding This work was supported with a research grant provided by Edwards Lifesciences (Nyon, Switzerland).

Competing interests PB is the representative of the IPPMed, Cloppenburg, Germany. NF, RPS, DM-Z and TKR are consultants to Edwards Lifesciences. The institutions of these three and those of the remaining authors representing study centres have received funding for employing a study nurse.

Patient consent for publication Not required.

Ethics approval The study was carried out in accordance with the Declaration of Helsinki and was approved by the independent ethics review board at each participating institution.

Provenance and peer review Not commissioned; externally peer reviewed.

Data availability statement Data are available on reasonable request. Data are available on reasonable request from the corresponding author.

Open access This is an open access article distributed in accordance with the Creative Commons Attribution Non Commercial (CC BY-NC 4.0) license, which permits others to distribute, remix, adapt, build upon this work non-commercially, and license their derivative works on different terms, provided the original work is properly cited, appropriate credit is given, any changes made indicated, and the use is non-commercial. See: http://creativecommons.org/licenses/by-nc/4.0/.

\section{ORCID iDs}

David Messika-Zeitoun http://orcid.org/0000-0002-6278-5670

Peter Bramlage http://orcid.org/0000-0003-4970-2110

\section{REFERENCES}

1 Osnabrugge RLJ, Mylotte D, Head SJ, et al. Aortic stenosis in the elderly: disease prevalence and number of candidates for transcatheter aortic valve replacement: a meta-analysis and modeling study. J Am Coll Cardiol 2013;62:1002-12.

2 lung $B$, Baron G, Butchart EG, et al. A prospective survey of patients with valvular heart disease in Europe: the Euro heart survey on valvular heart disease. Eur Heart J 2003;24:1231-43.

3 Ramaraj R, Sorrell VL. Degenerative aortic stenosis. BMJ 2008;336:550-5
4 Baumgartner H, Falk V, Bax JJ, et al. 2017 ESC/EACTS guidelines for the management of valvular heart disease. Eur Heart $J$ 2017;38:2739-91.

5 Malaisrie SC, Tuday E, Lapin B, et al. Transcatheter aortic valve implantation decreases the rate of unoperated aortic stenosis. Eur $J$ Cardiothorac Surg 2011;40:43-8.

6 Frey N, Steeds RP, Rudolph TK, et al. Symptoms, disease severity and treatment of adults with a new diagnosis of severe aortic stenosis. Heart 2019;105:1709-16.

7 Steeds RP, Lutz M, Thambyrajah J, et al. Facilitated data relay and effects on treatment of severe aortic stenosis in Europe. J Am Heart Assoc 2019;8:e013160.

8 Bach DS, Siao D, Girard SE, et al. Evaluation of patients with severe symptomatic aortic stenosis who do not undergo aortic valve replacement: the potential role of subjectively overestimated operative risk. Circ Cardiovasc Qual Outcomes 2009;2:533-9.

9 Frey N, Steeds RP, Serra A, et al. Quality of care assessment and improvement in aortic stenosis - rationale and design of a multicentre registry (IMPULSE). BMC Cardiovasc Disord 2017;17:5.

10 Fukui S, Kawakami M, Otaka Y, et al. Physical frailty in older people with severe aortic stenosis. Aging Clin Exp Res 2016;28:1081-7.

11 Clavel M-A, Magne J, Pibarot P. Low-gradient aortic stenosis. Eur Heart J 2016;37:2645-57.

12 Dalsgaard M, Kjaergaard J, Pecini R, et al. Predictors of exercise capacity and symptoms in severe aortic stenosis. Eur J Echocardiogr 2010;11:482-7.

13 Takeda S, Rimington H, Chambers J. Prediction of symptom-onset in aortic stenosis: a comparison of pressure drop/flow slope and haemodynamic measures at rest. Int J Cardiol 2001;81:131-7.

14 Briasoulis A, Bakris GL. Chronic kidney disease as a coronary artery disease risk equivalent. Curr Cardiol Rep 2013;15:340.

15 Fallen EL, Elliott WC, Gorlin R. Mechanisms of angina in aortic stenosis. Circulation 1967;36:480-8.

16 Fuchs C, Mascherbauer J, Rosenhek R, et al. Gender differences in clinical presentation and surgical outcome of aortic stenosis. Heart 2010;96:539-45.

17 Arnar DO. Syncope in patients with structural heart disease. J Intern Med 2013;273:336-44.

18 Bauer F, Coutant V, Bernard M, et al. Patients with severe aortic stenosis and reduced ejection fraction: earlier recovery of left ventricular systolic function after transcatheter aortic valve implantation compared with surgical valve replacement. Echocardiography 2013;30:865-70.

19 Mok M, Nombela-Franco L, Dumont E, et al. Chronic obstructive pulmonary disease in patients undergoing transcatheter aortic valve implantation: insights on clinical outcomes, prognostic markers, and functional status changes. JACC Cardiovasc Interv 2013;6:1072-84.

20 Roberts MM, Cho J-G, Sandoz JS, et al. Oxygen desaturation and adverse events during 6-min walk testing in patients with COPD. Respirology 2015;20:419-25.

21 Tarantini G, Mojoli M, Windecker S, et al. Prevalence and Impact of Atrial Fibrillation in Patients With Severe Aortic Stenosis Undergoing Transcatheter Aortic Valve Replacement: An Analysis From the SOURCE XT Prospective Multicenter Registry. JACC CardiovasC Interv 2016:9:937-46.

22 Stortecky S, Buellesfeld L, Wenaweser P, et al. Atrial fibrillation and aortic stenosis: impact on clinical outcomes among patients undergoing transcatheter aortic valve implantation. Circ Cardiovasc Interv 2013;6:77-84.

23 Moretti M, Fabris E, Morosin M, et al. Prognostic significance of atria fibrillation and severity of symptoms of heart failure in patients with low gradient aortic stenosis and preserved left ventricular ejection fraction. Am J Cardiol 2014;114:1722-8.

24 Katz S, Downs TD, Cash HR, et al. Progress in development of the index of ADL. Gerontologist 1970;10:20-30. 